\title{
Abdominal hollow viscus trauma in children, injury mechanisms and treatment principles
}

\author{
Elena Tarca ${ }^{1}$, Irina Ciomaga ${ }^{2}$, Nicolae Nistor ${ }^{2}$, Irina Criscov² ${ }^{2}$ Tamara Solange Rosu ${ }^{3}$ \\ ${ }^{1}$ Pediatric Surgery and Orthopedics Discipline, Surgical Department II, \\ "Gr. T. Popa" University of Medicine and Pharmacy, Iasi, Romania \\ ${ }^{2}$ Pediatrics Discipline, Mother and Child Medicine Department, \\ "Gr. T. Popa" University of Medicine and Pharmacy, Iasi, Romania \\ ${ }^{3}$ Nursing Discipline, Medical Department II, \\ "Gr. T. Popa" University of Medicine and Pharmacy, Iasi, Romania
}

\begin{abstract}
Abdominal hollow viscus trauma represents an important cause of pediatric morbidity, following head, vertebral column, spinal cord and thoracic injuries, whose main mechanisms of production are road traffic accidents, falls with abdominal impact, sports injuries, physical aggression and explosions. If there is a penetrating abdominal trauma, most frequently it associates hollow organ injuries, while abdominal blunt trauma is associated more often with parenchymal injuries, with effects on the hemodynamic status. Imaging and paraclinical investigations used to evaluate an abdominal trauma consist of thoraco-abdominal radiography in orthostatic position, abdominal ultrasonography, diagnostic peritoneal lavage, abdominal computed tomography and exploratory laparoscopy.

Unlike penetrating injuries, when surgical exploration is obvious, in the case of young children with abdominal contusions, the injuries involving hollow viscus can be overlooked at time of the initial assessment, because of a poor communication between doctor and patient, difficult interpretation of symptoms when there are other injuries associated, but also because of the intial lack of ultrasound signs of perforation. Delayed diagnosis and adequate treatment lead to extended duration of hospitalisation, to elevated costs and rates of morbidity. The incidence of mortality caused by abdominal trauma associated with hollow viscus penetration is approximately $10 \%$, being more elevated as the digestive injuries are multiple or associated with other lesions.
\end{abstract}

Keywords: accident, child, abdominal trauma, gastrointestinal perforation

\section{INTRODUCTION}

Abdominal hollow viscus trauma represents an important cause of pediatric morbidity, following head, vertebral column, spinal cord and thoracic injuries. The main mechanisms of production are road traffic accidents in the first place, fallowed by falls with abdominal impact, sports injuries, physical aggression and explosions. The number of pediatric patients admitted due to bike related injury has also been gradually increasing. Children who suffer from direct impact of the handlebars are more likely to require abdominal operative intervention and pediatric intensive care unit admission than those who fall (1). Compared to adults, chil- dren are more vulnerable to abdominal injuries, because they have thinner abdominal wall musculature, more flexible ribs and a relatively flat diaphragm. In penetrating abdominal trauma, hollow organs are more commonly involved, while abdominal blunt trauma is associated more often with solid organ injuries, with effects on hemodynamic status. Abdominal trauma is present in approximately $25 \%$ of pediatric patients with major trauma and is the most common cause of unrecognized fatal injury în children (2). A retrospective study including over 257,000 patients with blunt abdominal trauma proves an incidence of $0.3 \%$ when colic perforations are involved and $1.1 \%$ for small bowel ones. The main cause of this kind of 
injuries consists of road accidents, followed by physical aggression, falls from height, industrial or sport accidents and even Heimlich maneuver (gastric perforations when the stomach is full and the compression force is too powerful) (3).

\section{Mechanisms of abdominal organ injuries}

The traffic legislation, through the obligation of wearing the seat belt, played an important role in reducing mortality in road traffic collisions, but it increased seat belt-related injuries through deceleration mechanisms, compression and laceration forces (4).

There are multiple gastrointestinal air-tissue interfaces, which make it prone to injuries, caused by differences of acoustic impedance. At the time of the accident, a series of mechanisms are involved, such as acceleration-deceleration, compression or laceration forces, which are responsible for mesenteric injuries, ruptures of the aorta, solid or hollow organ injuries.

Direct compression forces against hollow viscus or external compression against a fixed object (seat belt, spinal column) lead to sudden increased intraluminal pressure and rupture caused by explosion on the antimesenteric border, while deceleration forces cause mesenteric or bowel tears. Less frequently, but still possible, visceral injuries can be caused by a pelvic fracture which perforates. Gastric and colic perforations will rapidly lead to peritonitis due to acidic juices and bacterial contamination, while the jejunal ones have less noisy symptoms due to alkaline content and low bacterial load. Furthermore, the symptoms can be influenced by the associated injuries, such as thoracic, cranial or spinal trauma, coma, hypovolemic shock.

Abdominal viscus trauma caused by road traffic accidents is well studied. Intestinal perforations are the third most frequent lesions, following hepatic and splenic ones, being revealed during laparotomies performed for abdominal contusions in approximately $5-16 \%$ of cases; rectal and gastric perforations occur less frequently, with an incidence that varies between 0.1 and $0.5 \%$, respectively $0.02-1.7 \%(5,6)$. The decreased frequency of gastric perforations caused by blunt abdominal trauma is due to the protection offered by the thoracic cage, increased mobility of viscus, but also to the gastric wall thickness. The most common location for gastric perforation is the anterior wall $(40 \%)$, followed by the greater curvature $(23 \%)$, lesser curvature $(15 \%)$ and posterior wall (15\%), being frequently associated with other injuries, such as splenic or thoracic ones (7). The duodenum, being a retroperitoneal organ, is rarely injured, the most frequent lesions parietal contusions or intramural hematoma.

The intramural hematoma can expand progressively and cause occlusion or ischemia, being at risk of perforation and secondary peritonitis. In very severe cases, there can appear intra or retroperitoneal duodenal ruptures, associated with pancreatic lesions, which can make a cephalic duodeno-pancreatectomy necessary. Gallbladder ruptures, caused by abdominal trauma, have been very rarely described in the medical literature, especially in adult cases, with an incidence that varies in the interval of $0.5 \pm 0.6 \%$ (8). Peritonitis syndrome occurring a few days after abdominal trauma may also be caused by an acute appendicitis due to both the trauma itself and the background of immune depression due to posttraumatic stress. Whatever the cause, post-traumatic peritonitis syndrome should be explored surgically (9).

\section{Principles of diagnosis and treatment}

Imaging and paraclinical investigations used to evaluate an abdominal trauma consist of thoracoabdominal radiography in orthostatic position, abdominal ultrasonography, diagnostic peritoneal lavage, abdominal computed tomography and exploratory laparoscopy.

Radiographic visualisation of pneumoperitoneum rapidly diagnoses hollow viscus perforation (Fig. 1), but the dynamic evaluation of a patient with negative initial radiological signs can reveal after 12-36 hours the presence of pneumoperitoneum or hydroaeric levels which are suggestive for secondary peritonitis. Abdominal ultrasonography is a non invasive, cheap and fast investigation, which requires neither the injection of a contrast medium, nor sedation, being at the same time very accurate in revealing abdominal traumatic lesions, especially involving the solid viscus, and also the free peritoneal liquid (10). Focused Assessment with Sonography for Trauma (FAST) can detect the presence of free fluid suggestive of intra-abdominal injury. In children, the utility of FAST is limited because less than half of pediatric patients with abdominal injury have free fluid (2). Also, if the patient is hemodynamically stable, the ultrasound finding of peritoneal free liquid does not establish the indication of laparotomy (it does not accurately identify the source of the fluid - blood or digestive liquid?); furthermore, the mesenteric and bowel lesions cannot be visualised with the help of the ultrasound. 


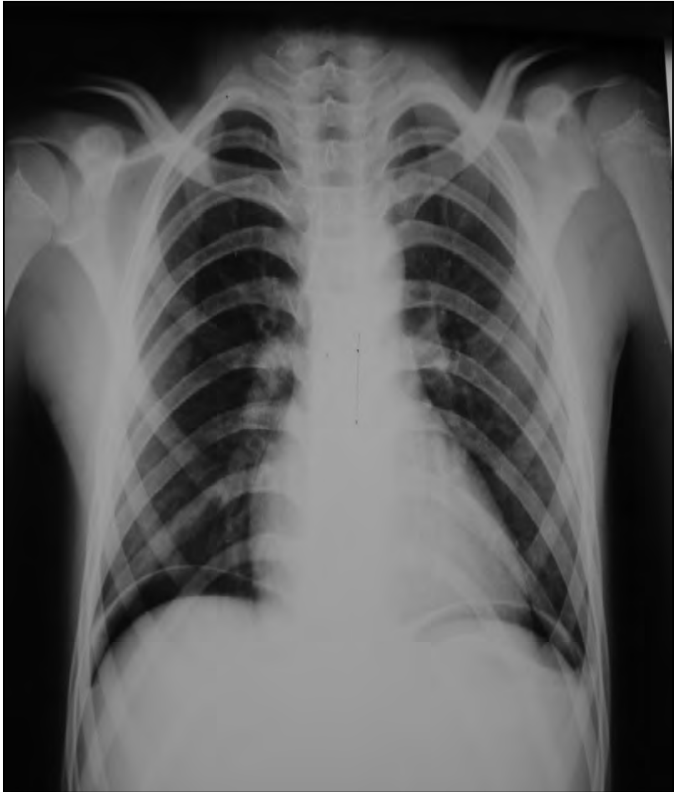

FIGURE 1. Pneumoperitoneum through cavitary organ perforation

With an accuracy of $86 \%$, a sensitivity of $94 \%$ and a predictivity of $92 \%$, abdominal computed tomography can detect intestinal and mesenteric injuries or perforations. The mesenteric trauma requires dynamic evaluation due to its unfavorable evolution in the next hours or days (11). However, the need for computed tomography scan may be obviated in a subset of low-risk pediatric blunt abdominal trauma patients presenting with a Glasgow Coma Scale of 14 to 15 , a normal abdominal examination result, and a negative FAST result (12). Diagnostic peritoneal puncture and lavage are simple maneuvers that can reveal a hemoperitoneum caused by solid viscus rupture or an intestinal perforation through the detection of intraperitoneal liquid, but they are not specific and more than that, they are invasive and less practicable for children who don 't cooperate, being replaced by abdominal computed tomography. Laparoscopic exploration has the advantage that it can accurately diagnose and, at the same time, can clean, wash and suture intestinal perforations with minimal bacterial contamination.

Abdominal pain and abdominal linear ecchymosis, especially in case of association with pelvic or lumbar spinal lesions (,seat belt syndrome“), represent an indication of exploration of the peritoneal cavity in order to detect viscus lesions, even if the other imaging investigations can appear normal. The term "seat belt syndrome" was first used and described in 1963 by Aiken (13). Furthermore, bicycle handlebar injury among children (Fig. 2) will indicate a high chance of abdominal viscus trauma.
Unlike penetrating injuries, when surgical exploration is obvious, in the case of young children with abdominal contusion, the injuries of hollow organs can be overlooked at the initial assessment, because of a poor communication between doctors and patients, difficult interpretation of symptoms when there are associated other injuries, but also because of the initial lack of ultrasound signs of perforation. Commonly seen in adults, but also in juvenile patients who are part of certain socially disadvantaged categories, acute poisoning with alcohol or other types of drugs may be the cause of the accident and may mask the painful posttraumatic symptoms (14). Delayed diagnosis and adequate treatment lead to an extended duration of hospitalization, elevated costs and rates of morbidity (15).

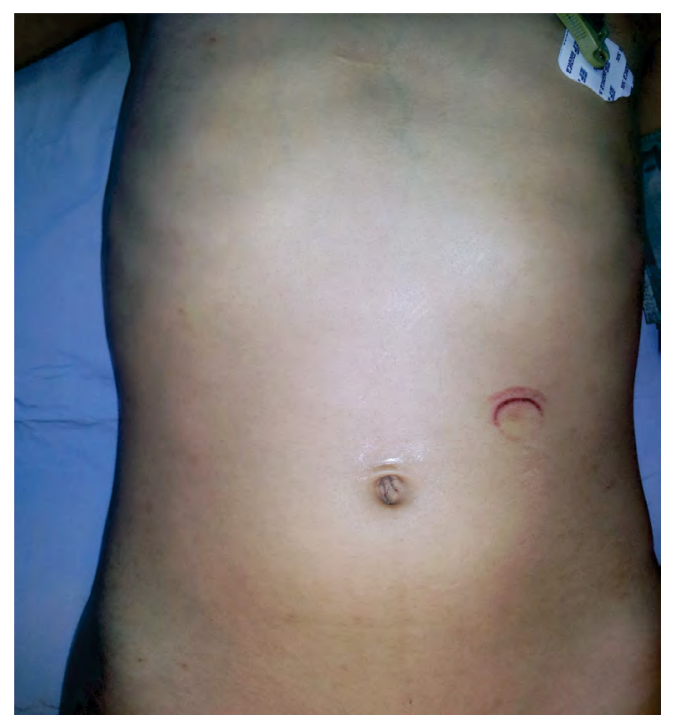

FIGURE 2. Traumatic mark (bicycle handlebars) jejunal perforation

Initial management of the trauma patient in shock includes fluid boluses of normal saline or Ringer's lactate with two, large-bore upper extremity catheters. Transfusion with packed red blood cells is done if the patient remains hypotensive after the second fluid bolus (2).

The management of cases with low intensity abdominal pain, hemodynamically stable and with normal imaging investigations includes hospitalization, repeated physical examinations and biological evaluations (hemogram, transaminases, inflammatory syndrome, lactate, urinalysis). Any status modification of the patient leads to imaging investigations repetition and even surgery, due to the fact that there are some possible lesions with late manifestation, such as parietal or mesenteric injuries which lead to ischemia and secondary perforation 
(16). Unlike the management of solid viscus injuries, which is conservative, the treatment of hollow viscus perforation consists of urgent surgery due to its risk of peritonitis and sepsis. The main aim of the surgical treatment consists of rapid control of the bleeding and peritoneal contamination source, with emphasis on the hydro-electrolytic and acidobasic resuscitation, correction of hypothermia and coagulopathy with the possibility of postponing the anatomical reestablishment if the patient's status is severely influenced (17).

Duodenal, jejunal and ileal unique perforations will be sutured in a single plane, while the gastric, colorectal and bladder ones will need dual plane sutures. Intestinal resections and anastomoses can be necessary if the lesions are extended or multiple; for colic injuries it is sometimes safer to use a stoma placed above the anastomosis. Antibiotic treatment is compulsory. Preventing abdominal hypertension and abdominal compartment syndrome due to extended retroperitoneal hematomas or to other viscus lesions which need a second-look is possible through the open abdomen management (laparostomy), but especially through using a vacuum-like system which proved its efficiency and even improved the long term survival rates (18).

Bladder perforations in children are rare, with an incidence between 0.05 and $2.0 \%$ of all pelvic traumas, being frequently associated with pelvic fractures (19). At very young ages the bladder and the stomach are located intraperitoneal, both of them using a narrow orifice which cannot provide a rapid elimination and consequently being at risk of explosion at the moment of sudden compression between two rigid plans. The bladder dome, being the weakest, is most commonly affected, resulting in intraperitoneal urinary extravasation. The first sign of bladder perforation consists of hematuria, besides other signs of abdominal contusion (traumatic mark, abdominal distension, peritonitis in case of association of digestive perforation). Sometimes an abdominal or pelvic penetrating injury can extend to the bladder, this being the reason why it is mandatory to use general anesthesia while exploring (Fig. 4). The diagnosis is confirmed by ultrasonography, cystoscopy, cystography or computed tomography. Intraperitoneal perforations are treated with dual plane sutures done laparoscopically or through a conservative aboard. As far as extraperitoneal perforations are concerned, urethral or suprapubic drainage can be enough.

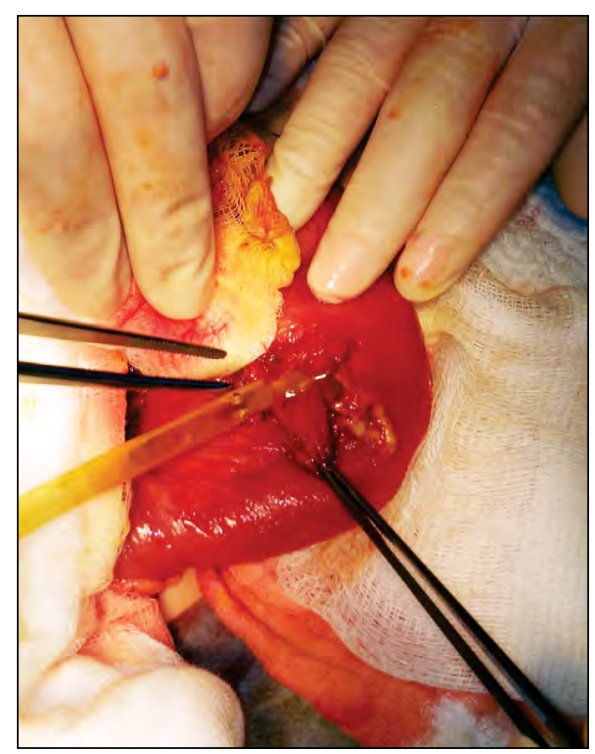

FIGURE 3. The jejunal perforation in the patient of Figure 2

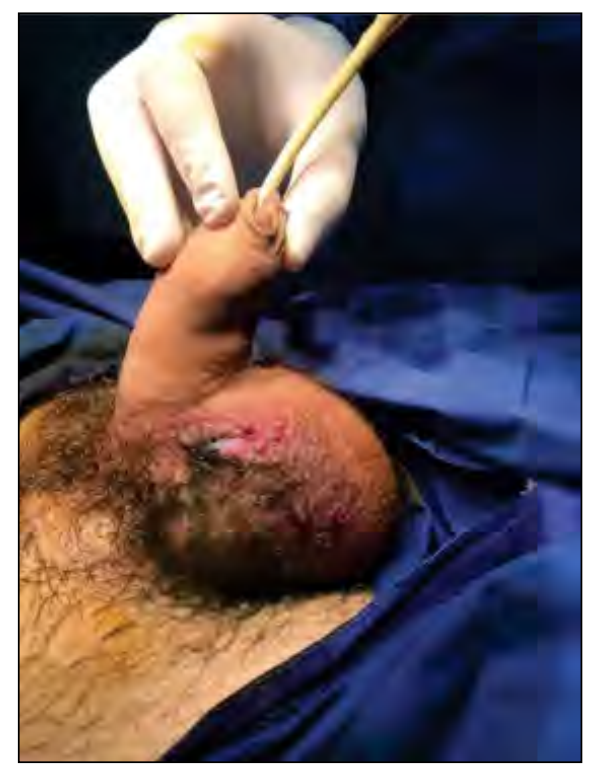

FIGURE 4. Scrotal wound removing the methyl blue inserted into the urine probe (bladder perforation)

Mortality rate for abdominal trauma associated with hollow organ perforations is approximately $10 \%$, being the highest when the injuries are multiple or associated with other lesions $(3,20)$. The highest morbidity and mortality rate occurs in gastric perforations, increasing parallel with the time passed to surgery: intervention within 2 hours leads to $2 \%$ mortality, while after 24 hours to over $30 \%$, due to traumatic lesions associated with septic shock (21).

\section{CONCLUSIONS}

Pediatric abdominal hollow viscus trauma represents a pathology with an increasing frequency and severity due to the rapid development of civil 
society, road traffic accidents being the most common cause. To reduce to a minimum the morbidity mechanism, to diagnose and anticipate the evoluand mortality, it is essential to know the injury

tion of the abdominal viscus injury, and more important, a rapid and adequate treatment involving a multidisciplinary team in case of association of orthopedic and neurosurgical lesions is essential.

Conflict of interest: none declared Financial support: none declared

\section{REFERENCES}

1. Klin B, Efrati Y, Vaiman M, Kozer E, Jeroukhimov I, Abu-Kishk I. Abdominal injuries following bicycle-related blunt abdominal trauma in children. Minerva Pediatr. 2016;68(3):167-72.

2. Lynch T, Kilgar J, Al Shibli A. Pediatric Abdominal Trauma. Curr Pediatr Rev. 2018;14(1):59-63.

3. Watts DD, Fakhry SM. EAST Multi-Institutional Hollow Viscus Injury Research Group. Incidence of hollow viscus injury in blunt trauma: an analysis from 275,557 trauma admissions from the East multiinstitutional trial. J Trauma. 2003;54(2):289-294.

4. Abbas AK, Hefny AF, Abu-Zidan FM. Seatbelts and road traffic collision injuries. World J Emerg Surg 2011; 6: 18.

5. Cripps NP, Cooper GJ. Intestinal injury mechanisms after blunt abdominal impact. Ann R Coll Surg Engl 1997; 79: 115-120.

6. Williams MD, Watts $D$, Fakhry $S$. Colon injury after blunt abdominal trauma: Results of the EAST Multi-Institutional Hollow Viscus Injury Study. J Trauma 2003; 55: 906-912.

7. M.R. Aboobakar, J.P. Singh, K. Maharaj et al. Gastric perforation following blunt abdominal trauma. Trauma Case Reports 10. 2017; 12-15.

8. Losanoea JE, Kjossev KT. Complete traumatic avulsion of the gallbladder. Injury, Int. J. Care Injured.1999; 30: 365-368.

9. Tarcă E, Gavrilescu S. Etiological and clinical-paraclinical implications of acute abdominal pain in children. Rev. Med. Chir. Soc. Med. Nat. 2017; 121(3): 568-576.

10. Nural MS, Yardan T, Güven $\mathrm{H}$ et al. Diagnostic value of ultrasonography in the evaluation of blunt abdominal trauma. Diagn Interv Radiol. 2005;11(1):41-44.

11. Killeen KL, Shanmuganathan K, Poletti PA et al. Helical computed tomography of bowel and mesenteric injuries. J Trauma. 2001;51(1):26-36.
12. Liang T, Roseman E, Gao M, Sinert R. The Utility of the Focused Assessment With Sonography în Trauma Examination in Pediatric Blunt Abdominal Trauma: A Systematic Review and Meta-Analysis. Pediatr Emerg Care. 2019 Mar 12.

13. Aiken DW. Intestinal perforation and facial fracture in an automobile acci-dent victim wearing a seat belt. Journal of the Louisiana State Medical Society 1963;115:235.

14. Roşu VE, Tarcă E, Bădărău OL, Roşu ST. "Binge drinking": Clinical and social aspects in pediatric emergencies - Case presentation. Romanian Journal of Oral Rehabilitation. 2019; 11(1): 121-124.

15. Clendenon JN, Meyers RL, Nance ML et al. Management of duodenal injuries in children. J Pediatr Surg 2004;39:964-968.

16. Biswas S, Adileh M, Almogy G, Bala M. Abdominal injury patterns in patients with seatbelt signs requiring laparotomy. J Emerg Trauma Shock 2014; 7: 295-300.

17. Loveland JA, Boffard KD. Damage control in the abdomen and beyond. Br J Surg 2004; 91: 1,095-1,101.

18. Cheatham ML, Demetriades D, Fabian TC et al. Prospective study examining clinical outcomes associated with a negative pressure wound therapy system and Barker's vacuum packing technique. World J Surg 2013; 37: 2018- 2030.

19. Dokucu Al, Ozdemir E, Oztürk H et al. Urogenital injuries in childhood: A strong association of bladder trauma to bowel injuries. Int Urol Nephrol. 2000;32:3-8.

20. Burch JM. Injury to the colon and rectum. In: Moore EE, Feliciano DV, Mattox KL (eds) Trauma, 5th ed. McGraw Hill, New York. 2004; 735-753.

21. R Maheshwari, A Sayana, $P$ Mahesh. Gastric rupture following blunt trauma abdomen: A case report. Indian J. Clin. Pract. 2013; 23 (12):859-860. 